\title{
Joint time-frequency diversity for single-carrier block transmission in frequency selective channels
}

\author{
Jinsong WU ${ }^{\dagger}$, Nonmember, Steven D. BLOSTEIN ${ }^{\dagger \dagger}$, Member, Qingchun CHEN ${ }^{\dagger \dagger}$, Nonmember, \\ and Pei XIAO ${ }^{\dagger+\dagger}$, Member
}

\begin{abstract}
SUMMARY In time-varying frequency selective channels, to obtain high-rate joint time-frequency diversity, linear dispersion coded orthogonal frequency division multiplexing (LDC-OFDM), has recently been proposed. Compared with OFDM systems, single-carrier systems may retain the advantages of lower PAPR and lower sensitivity to carrier frequency offset (CFO) effects, which motivates this paper to investigate how to achieve joint frequency and time diversity for high-rate single-carrier block transmission systems. Two systems are proposed: linear dispersion coded cyclic-prefix single-carrier modulation (LDC-CP-SCM) and linear dispersion coded zero-padded single-carrier modulation (LDC-ZP-SCM) across either multiple CP-SCM or ZP-SCM blocks, respectively. LDCSCM may use a layered two-stage LDC decoding with lower complexity. This paper analyzes the diversity properties of LDC-CP-SCM, and provides a sufficient condition for LDC-CP-SCM to maximize all available joint frequency and time diversity gain and coding gain. This paper shows that LDC-ZP-SCM may be effectively equipped with low-complexity minimum mean-squared error (MMSE) equalizers. A lower complexity scheme, linear transformation coded SCM (LTC-SCM), is also proposed with good diversity performance.

key words: linear dispersion codes, OFDM, diversity order, frequency domain equalization, cyclic-prefix, zero-padding, carrier frequency offsets, MMSE, low complexity, frequency selective channels, LDC-SCM, LTC$S C M$
\end{abstract}

\section{Introduction}

With the increase of data rates, broadband communication signals often experience frequency selective fading. Recently, cyclic-prefix single-carrier modulation (CP-SCM) with frequency-domain equalization (FDE) has attracted a lot of attention [1-3]. Similar to orthogonal frequencydivision multiplexing (OFDM) [4], CP-SCM FDE provides much lower computational complexity than conventional time-domain equalization techniques, especially for long impulse response tail channels [1]. Unlike OFDM, CP-SCM does not suffer high peak-to-average power ratio (PAPR) as well as sensitivity to frequency and phase offsets (carrierfrequency offsets, or CFO) [4], and nonlinear distortions [5]. The complexity of CP-SCM-FDE transmitter is simpler than that of OFDM, due to lack of Fast Fourier Transform (FFT) components, and [1] encourages the use of CP-SCM in the

\footnotetext{
${ }^{\dagger}$ Jinsong Wu is with Bell laboratories, Shanghai, P.R. China, 201206, email: wujs@ieee.org

${ }^{\dagger}$ Steven D. Blostein is with Queen's University, Kingston, Ontario, Canada, email: steven.blostein@ queensu.ca

Q Qingchun Chen is with School of Information Science \& Technology, Southwest Jiaotong University, Chengdu, P.R. China, 610031, email: qcchen@home.swjtu.edu.cn

${ }^{t+\dagger \dagger}$ Pei Xiao is with University of Surrey, UK, email: P.Xiao@surrey.ac.uk

DOI: $10.1587 /$ transfun.E0.A.1
}

uplink and OFDM in the downlink in order to reduce the processing complexity at the terminal.

In [6], high-rate linear dispersion codes have been employed to obtain joint frequency and time diversity in OFDM, known as LDC-OFDM. Although LDC has been applied to multicarrier communications, limited efforts have been devoted to investigating LDC in single stream single carrier communications. This paper investigates the application of LDC to achieve joint frequency-time diversity in CP-SCM over frequency selective channels.

This paper proposes to apply linear dispersion codes to single-carrier block communications (SCBC). Two types of SCBC are considered: cyclic-prefix single-carrier modulation (CP-SCM) and zero-padded single-carrier modulation (ZP-SCM). CP-SCM utilizes frequency-domain equalization (FDE) with lower complexity, due to its use of the computationally-efficient fast Fourier transform (FFT). Note that the complexity of time-domain equalization (TDE) using Viterbi algorithms grows exponentially with channel memory and spectral efficiency (trellis-based schemes) or requires very long FIR filters to achieve satisfactory performance (e.g., decision feedback equalizers) [1]. This paper provides and proves the sufficient condition for LDC-CP$\mathrm{SCM}$ to maximize the joint frequency-time diversity gain. Moreover, this paper shows that ZP-SCM may have lower complexity using approximate frequency-domain equalization (AFDE). Both CP-SCM and ZP-SCM enjoy lower $\mathrm{PAPR}$ and are more robust to $\mathrm{CFO}[1,7]$.

\section{Single-carrier communications model}

The communications channel is assumed to experience frequency-selective fading, and the channel for the $k$-th SCM block is modeled as an $L$ th-order FIR filter with impulse response $\mathbf{h}^{(k)}=\left[h_{0}^{(k)}, \ldots, h_{L}^{(k)}\right]^{T}$. Channel coefficients are assumed to be constant within one SCM block but change statistically independently across different SCM blocks. Each SCM block is of size $P=N_{C}+N_{g}$, including a data symbol block of size $N_{C}$ and a guard interval of size $N_{g} \geq L$ to avoid inter-block interferences.

Denote $\mathbf{x}_{S C}^{(k)}$ as the channel data symbol vector transmitted during the $k$-th SCM block of size $N_{C} \times 1$, and $\mathbf{x}_{S C}^{(k)}=\left[x_{S C(1)}^{(k)}, \ldots, x_{S C\left(N_{C}\right)}^{(k)}\right]^{\mathcal{T}}$, where $x_{S C(p)}^{(k)}, p=1, \ldots, N_{C}$ is the $p$-th data symbol of the $k$-th SCM block in sequence. Each receive antenna experiences additive white complex 
Gaussian noise. The system signal-to-noise-ratio (SNR) is denoted by $\rho$.

\subsection{CP-SCM case}

Before transmission, a cyclic prefix (CP) guard interval is appended to each CP-SCM block. The CP is then removed at the receiver. The effective channel of the $k$-th SCM block is a circulant matrix $\mathbf{H}_{C P S C}^{(k)}$ with elements $\left[\mathbf{H}_{C P S C}^{(k)}\right]_{a, b}=$ $h_{\left((a-b) \bmod N_{C}\right)}^{(k)}$. Hence, the CP-SCM block system can be modeled as

$$
\mathbf{r}_{C P \_S C}^{(k)}=\sqrt{\rho} \mathbf{H}_{C P \_S C}^{(k)} \mathbf{x}_{S C}^{(k)}+\mathbf{v}_{C P \_S C}^{(k)}
$$

where $\mathbf{r}_{C P S C}^{(k)}$ is the received block after CP removal, and $\mathbf{v}_{C P S C}^{(k)}$ is the corresponding noise vector.

At the receiver, the received block $\mathbf{r}_{C P S C}^{(k)}$ is first processed by an FFT to generate block $\mathbf{y}_{C P S C}^{(k)}=\mathbf{F}_{N_{C}} \mathbf{r}_{C P S C}^{(k)}$, where $\mathbf{F}_{K}$ denote the discrete Fourier transform (DFT) matrix, representing the $K$-point fast Fourier transform (FFT) with entries, $\left[\mathbf{F}_{K}\right]_{a, b}=(1 / \sqrt{K}) \exp (-j 2 \pi(a-1)(b-1) / K)$.

Due to the circulant property, $\mathbf{H}_{C P \_S C}^{(k)}$ can be decomposed as

$$
\mathbf{H}_{C P S C}^{(k)}=\left[\mathbf{F}_{N_{C}}\right]^{H} \mathbf{D}_{C P S C}^{(k)} \mathbf{F}_{N_{C}},
$$

where $\mathbf{D}_{C P S C}^{(k)}$ is diagonal with

$$
\left[\mathbf{D}_{C P \_S C}^{(k)}\right]_{p p}=\sum_{l=0}^{L} h_{l}^{(k)} \exp \left(-j 2 \pi l(p-1) / N_{C}\right) .
$$

Thus, the frequency domain system equation is

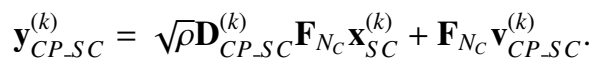

\subsection{ZP-SCM case}

Due to zero padding, the ZP-SC system model does not have a simple frequency domain format shown in (2). However, the ZP-SCM system model can be written in block matrix form in the time domain as,

$$
\mathbf{r}_{Z P_{-} S C}^{(k)}=\sqrt{\rho} \mathbf{H}_{Z P_{-} S C}^{(k)} \mathbf{x}_{S C}^{(k)}+\mathbf{v}_{Z P_{-} S C}^{(k)},
$$

where $\mathbf{H}_{Z P S C}^{(k)}$ represents a Toeplitz convolution matrix with $\left[\mathbf{H}_{Z P_{-} S C}^{(k)}\right]_{a, b}=h_{(a-b)}^{(k)}$, where $\mathbf{r}_{Z P_{-} S C}^{(k)}$ is the received block of size $P \times 1$, and $\mathbf{v}_{Z P S C}^{(k)}$ is the corresponding noise vector. Due to the Toeplitz structure, $\mathbf{H}_{Z P-S C}^{(k)}$ is guaranteed to be invertible, regardless of the channel zero locations in reality [8].

\section{LDC Encoding}

It is assumed that an uncorrelated data sequence has been modulated using complex-valued source data symbols chosen from an arbitrary, e.g. r-PSK or r-QAM, constellation. A $T \times M$ LDC matrix codeword, $\mathbf{S}_{L D C}$, is transmitted over
$M$ channels and occupies $T$ channel uses and encodes $Q$ source data symbols. Denote the LDC codeword matrix as $\mathbf{S}_{L D C} \in C^{T \times M}$, and $\mathbf{A}_{q} \in C^{T \times M}, \mathbf{B}_{q} \in C^{T \times M}, q=1, \ldots, Q$ are called dispersion matrices. Unlike [9], which considers LDC only as space time codes, we consider LDC as a general complex matrix coding framework.

Just as in [6, 10], we consider the case $\mathbf{A}_{q}=\mathbf{B}_{q}, q=$ $1, \ldots, Q$. We have LDC encoding in the matrix form equation,

$$
\operatorname{vec}\left(\mathbf{S}_{L D C}\right)=\mathbf{G}_{L D C} \mathbf{S},
$$

where $\mathbf{s}=\left[\begin{array}{lll}s_{1} & \cdots & s_{Q}\end{array}\right]^{T}$ is the source complex symbol vector,

$$
\mathbf{G}_{L D C}=\left[\operatorname{vec}\left(\mathbf{A}_{1}\right), \ldots, \operatorname{vec}\left(\mathbf{A}_{Q}\right)\right]
$$

is the LDC encoding matrix. Details can be found in $[6,10]$.

\section{Proposed LDC based single-carrier block communi- cations}

\subsection{Proposed system structure}

The proposed system model of LDC-CP-SCM is illustrated in Figure A 1 . One LDC codeword is across $T$ SCM blocks. The $T$ SCM blocks include $D$ LDC codewords with sizes of $T \times N_{F_{(i)}}$, where $N_{C}=\sum_{i=1}^{D} N_{F_{(i)}}$. Denote the LDC encoding matrix of the $i$-th LDC matrix codeword $\mathbf{S}_{L D C}^{(i)} \in C^{T \times N_{F_{(i)}}}$ as $\mathbf{G}_{L D C}^{(i)}$, which encodes source data symbol vector with zero mean, unit variance, $\mathbf{s}^{(i)}=\left[\begin{array}{llll}s_{1}^{(i)} & s_{1}^{(i)} & \cdots & s_{Q_{i}}^{(i)}\end{array}\right]^{\mathcal{T}}$ into $\operatorname{vec}\left(\mathbf{S}_{L D C}^{(i)}\right)$, where $Q_{i}$ is the number of source data symbols in $\mathbf{s}^{(i)}, i=1, \ldots, D$.

For the LDC-CP-SCM receiver, frequency-domain equalization (FDE) can be applied as illustrated in Figure A. 1. The received SCM data block is first FFT-processed. Then, the influence of the frequency-selective channel impulse response is eliminated by the FDE. The inverse FFT operation returns the equalized signals to the time domain prior to LDC decoding.

\subsection{LDC-SCM receiver}

\subsubsection{First estimation step - SCM demodulation}

In the proposed LDC decoding algorithm, LDC decoding is independent of SCM signal estimation. Thus, the proposed LDC-SCM system is backwards-compatible to conventional SCM systems.

In Section 6, performance is investigated using minimum-mean-squared-error (MMSE) equalization. Assuming that single carrier symbols are normalized to unit variance, the respective frequency and time domain equalizers are given by [11]

\section{CP-SCM MMSE-FDE}




$$
\begin{aligned}
& \mathbf{G}_{C P \_S}^{(k)}=\sqrt{\rho} \mathbf{C}_{\mathbf{x}_{S C}^{(k)}}\left(\mathbf{D}_{C P-S C}^{(k)}\right)^{\mathcal{H}} \cdot \\
& \left(\mathbf{I}_{N_{C}}+\rho \mathbf{D}_{C P-S C}^{(k)} \mathbf{C}_{\mathbf{x}_{S C}^{(k)}}\left(\mathbf{D}_{C P-S C}^{(k)}\right)^{\mathcal{H}}\right)^{-1} . \\
& \widehat{\mathbf{x}_{S C}^{(k)}}=\left[\mathbf{F}_{N_{C}}\right]^{\mathcal{H}} \mathbf{G}_{C P S C}^{(k)} \mathbf{y}_{C P-S C}^{(k)}
\end{aligned}
$$

\section{ZP-SCM MMSE-TDE}

$$
\begin{aligned}
& \mathbf{G}_{Z P \_S C}^{(k)}=\sqrt{\rho} \mathbf{C}_{\mathbf{x}_{S C}^{(k)}}\left(\mathbf{H}_{Z P-S C}^{(k)}\right)^{\mathcal{H}} \cdot \\
& \left(\mathbf{I}_{P}+\rho \mathbf{H}_{Z P-S C}^{(k)} \mathbf{C}_{\mathbf{x}_{S C}^{(k)}}\left(\mathbf{H}_{Z P-S C}^{(k)}\right)^{\mathcal{H}}\right)^{-1} . \\
& \widehat{\mathbf{x}_{S C}^{(k)}}=\mathbf{G}_{Z P S S C}^{(k)} \mathbf{r}_{Z P_{-} S C}^{(k)}
\end{aligned}
$$

where $k=1, \ldots, T, \mathbf{C}_{\mathbf{x}_{S C}^{(k)}}$ is the covariance matrix of the $k$ th SCM block within one LDC-SCM block, which can be calculated using LDC encoding matrices.

In ZP-SCM system, the carrier frequency offset (CFO) acts as multiplicative noise that reduces the useful signal amplitude but does not cause ISI, thus ZP-SCM is more robust against CFO. Although ZP-SCM system does not perform explicit accurate frequency domain equalization (FDE), ZP-SCM could be formulated as an approximate frequency domain equalizer (AFDE).

Denote

$$
\mathbf{D}_{\mathbf{H}_{P}^{(k)}}=\mathbf{F}_{P} \mathbf{H}_{P}^{(k)}\left[\mathbf{F}_{P}\right]^{\mathcal{H}}
$$

where $\mathbf{H}_{P}^{(k)}$ is a is a circulant matrix with $\left[\mathbf{H}_{P}^{(k)}\right]_{a, b}=$ $h_{\left((a-b) \bmod N_{C}\right)}^{(k)}$. Denote

$$
\mathbf{T}_{Z P}=\left[\begin{array}{ll}
\mathbf{I}_{N_{C}} & \mathbf{0}_{\left(P-N_{C}\right) \times N_{C}}
\end{array}\right]^{\mathcal{T}}
$$

and

$$
\mathbf{U}=\mathbf{F}_{P} \mathbf{T}_{Z P}
$$

Thus, we have

$$
\mathbf{H}_{Z P_{-} S C}^{(k)}=\left[\mathbf{F}_{P}\right]^{\mathcal{H}} \mathbf{D}_{\mathbf{H}_{P}^{(k)}} \mathbf{F}_{P} \mathbf{T}_{Z P}
$$

Consequently, we could rewrite (3) in the AFDE form,

$$
\mathbf{y}_{Z P_{-} S C}^{(k)}=\sqrt{\rho} \mathbf{D}_{\mathbf{H}_{P}^{(k)}} \mathbf{U} \mathbf{x}_{S C}^{(k)}+\mathbf{F}_{P} \mathbf{v}_{Z P_{-} S C}^{(k)}
$$

where

$$
\mathbf{y}_{Z P S C}^{(k)}=\mathbf{F}_{P} \mathbf{r}_{Z P \_S C}^{(k)} .
$$

The corresponding MMSE-AFDE equalizer is given by

$$
\begin{aligned}
& \mathbf{G}_{Z P S C}^{(k)}=\sqrt{\rho} \mathbf{U} \mathbf{C}_{\mathbf{x}_{S C}^{(k)}} \mathbf{U}^{\mathcal{H}}\left(\mathbf{D}_{\mathbf{H}_{P}^{(k)}}\right)^{\mathcal{H}} . \\
& \left(\mathbf{I}_{P}+\rho \mathbf{D}_{\mathbf{H}_{P}^{(k)}} \mathbf{U} \mathbf{C}_{\mathbf{x}_{S C}^{(k)}} \mathbf{U}^{\mathcal{H}}\left(\mathbf{D}_{\mathbf{H}_{P}^{(k)}}\right)^{\mathcal{H}}\right)^{-1} .
\end{aligned}
$$

Note that matrix inversion of the CP-SCM MMSE-FDE equalizer (6) may be performed element-wise if channel data symbols are uncorrelated. However, matrix inversion of ZP-SCM MMSE time domain equalization (MMSE-TDE) equalizer (9) cannot be accurately performed in the elementwise fashion due to the non-diagonal matrix $\mathbf{U}$. However, if $\mathbf{C}_{\mathbf{x}_{S C}^{(k)}}=\mathbf{I}_{N_{C}}$, using

$$
\mathbf{U U}^{\mathcal{H}} \approx \frac{N_{c}}{P} \mathbf{I}_{P},
$$

(9) can be approximated as

$$
\mathbf{G}_{Z P_{\perp} S C}^{(k)}=\sqrt{\rho}\left(\mathbf{D}_{\mathbf{H}_{P}^{(k)}}\right)^{\mathcal{H}} \cdot\left(\frac{P}{N_{C}} \mathbf{I}_{P}+\rho \mathbf{D}_{\mathbf{H}_{P}^{(k)}}\left(\mathbf{D}_{\mathbf{H}_{P}^{(k)}}\right)^{\mathcal{H}}\right)^{-1}
$$

which we call MMSE low complexity approximate FDE (MMSE-LC-AFDE). The approximation (10) may work better when $\frac{P-N_{c}}{P}$ is closer to zero, and in this case, $\mathbf{U}$ is closer to a discrete Fourier transform matrix. In (11), we extend the low complexity MMSE equalizer structure for ZP-OFDM in [8] to ZP-SCM systems.

\subsubsection{Second estimation step - LDC-SCM block decoding}

Reorganizing the results of the first estimation step into $D$ estimated LDC matrix codewords, $\widehat{\mathbf{S}_{L D C}^{(i)}}, i=1, \ldots, D$, the estimated data symbol vectors corresponding to $D$ LDC blocks are

$$
\widehat{\mathbf{s}^{(i)}}=\left[\mathbf{G}_{L D C}^{(i)}\right]^{\dagger} \operatorname{vec}\left(\widehat{\mathbf{S}_{L D C}^{(i)}}\right) .
$$

\subsection{Low complexity approaches - LTC-SCM}

Unlike the general class of LDC of codeword size $T \times M$, where $M>1$, we consider a special class of LDC of codeword size $T \times 1$, which we term linear transformation codes (LTC). Since single-carrier block communications themselves may achieve a certain degree of frequency diversity order, we proposes to apply LTC across multiple SCM blocks, which we call LTC-SCM. LTC-SCM is a class of low complexity approaches to achieve joint frequency and time diversity. Note that frequency diversity is not obtained from this transformation but from inherent properties of single-carrier block communications.

\subsection{Peak-to-average power ratio (PAPR)}

Single-carrier complex matrix codes (SCCMC) are currently proposed as space time block codes in the literature, and usually possess lower PAPR than OFDM. However, the PAPR of SCCMC is often higher than that of conventional constellation-based SCM. Fortunately, designing lower PAPR SCCMC is easier than designing lower PAPR OFDM based systems. Some initial efforts in addressing this issue can be found in $[12,13]$.

\subsection{Carrier frequency offsets}

Conventional constellation-based SCM have fewer problems with regard to carrier frequency offsets (CFO). We are 
interested in investigating performance of LDC-CP-SCM under CFO effects. With minor modification, we extend CPOFDM CFO system model in [14] to CP-SCM as follows:

$$
\begin{aligned}
& \mathbf{y}_{C P S C}^{(k)}=\sqrt{\rho} \phi(a) \mathbf{U}_{C F O} \mathbf{D}_{C P S C}^{(k)} \mathbf{F}_{N_{C}} \mathbf{x}_{S C}^{(k)} \\
& +\mathbf{F}_{N_{C}} \mathbf{v}_{C P S C}^{(k)},
\end{aligned}
$$

where

1. $\varepsilon=\Delta f T_{s} N_{C}$ is normalized CFO, $\Delta f$ is $\mathrm{CFO}$ and $T_{s}$ is the channel symbol period;

2.

$$
\phi(a)=\exp \left(j 2 \pi \varepsilon\left((a-1) P+N_{g}\right) / N_{C}\right)
$$

and

$$
a=\left\{\begin{array}{c}
k\left(\bmod N_{F S}\right) \text { if } k\left(\bmod N_{F S}\right) \neq 0 \\
N_{F S} \text { if } k\left(\bmod N_{F S}\right)=0 ;
\end{array}\right.
$$

where $N_{F S}$ stands for frequency synchronization rate (FSR) per SCM block. In other words, $(a-1)$ is set to zero every $N_{F S} \mathrm{SCM}$ blocks;

3. $\mathbf{U}_{C F O}=\mathbf{F}_{N_{C}} \mathbf{D}_{C F O}\left[\mathbf{F}_{N_{C}}\right]^{\mathcal{H}}$, where

$$
\begin{aligned}
& \mathbf{D}_{C F O}= \\
& \operatorname{diag}\left(\exp \left(j 2 \pi \varepsilon\left(1 / N_{C}\right)\right), \ldots, \exp \left(j 2 \pi \varepsilon\left(N_{C} / N_{C}\right)\right)\right) .
\end{aligned}
$$

For comparison purposes, we also consider CP-OFDM under $\mathrm{CFO}$ effects, i.e.,

$$
\begin{aligned}
& \mathbf{y}_{C P \_O F D M}^{(k)}= \\
& \sqrt{\rho} \phi(a) \mathbf{U}_{C F O} \mathbf{D}_{C P \_O F D M}^{(k)} \mathbf{x}_{O F D M}^{(k)} \\
& +\mathbf{F}_{N_{C}} \mathbf{v}_{C P \_O F D M}^{(k)} .
\end{aligned}
$$

Based on models (13) and (14), a comparison of CFO effects is provided through simulations in Section 6.3. Note that there are well-known techniques to reduce CFO effects using CFO compensation and estimation $[2,15,16]$. The CFO compensation for LDC-SCM may be considered as future work,

\section{Diversity properties}

For simplicity, we only analyze the diversity properties of LDC-CP-SCM, since it is easier to consider frequencydomain signals in order to study both temporal and frequency diversity, the chosen object to be analyzed is $\mathbf{z}_{C P-S C}^{(k)}=\mathbf{F}_{N c} \mathbf{x}_{S C}^{(k)}, k=1, \ldots, T$. Thus the whole LDC-CPSCM block with FFT outer processing in each SCM block can expressed as matrix $\mathbf{C}$

$$
\mathbf{C}=\left[\begin{array}{cccc}
c_{1}^{(1)} & c_{2}^{(1)} & \cdots & c_{N_{C}}^{(1)} \\
c_{2}^{(2)} & c_{2}^{(2)} & \cdots & c_{N_{C}}^{(2)} \\
\vdots & \vdots & \ddots & \vdots \\
c_{1}^{(T)} & c_{2}^{(T)} & \cdots & c_{N_{C}}^{(T)}
\end{array}\right],
$$

where $c_{p}^{(k)}=\left[\mathbf{z}_{C P-S C}^{(k)}\right]_{p, 1}, p=1, \ldots, N_{C}, k=1, \ldots T$.

We write the system equation for the block $\mathbf{C}$ as

$$
\mathbf{R}=\sqrt{\rho} \mathbf{M H}+\mathbf{V},
$$

where received signal vector $\mathbf{R}$ and noise vector $\mathbf{V}$ are of size $N_{C} T \times 1$. The chosen frequency symbol diagonal matrix $\mathbf{M}$ is of size $N_{C} T \times N_{C} T$, and

$$
\mathbf{M}=\operatorname{diag}\left(c_{1}^{(1)}, \ldots, c_{N_{C}}^{(1)}, \ldots, c_{1}^{(T)}, \ldots, c_{N_{C}}^{(T)}\right) .
$$

The channel vector $\mathbf{H}$ is of size $N_{C} T \times 1$, and

$$
\mathbf{H}=\left[H_{1}^{(1)}, H_{2}^{(1)}, \ldots, H_{N_{C}}^{(1)}, \ldots, H_{1}^{(T)}, H_{2}^{(T)}, \ldots, H_{N_{C}}^{(T)}\right]^{\mathcal{T}},
$$

where $H_{p}^{(k)}$ is the $p$-th subchannel gain of $k$-th SCM block in $\mathbf{C}$ in the frequency domain. Thus $H_{p}^{(k)}=\left[\mathbf{w}_{p}\right]^{T} \mathbf{h}^{(k)}$, where

$$
\mathbf{w}_{p}=\left[1, \omega^{p-1}, \omega^{2(p-1)}, \cdots, \omega^{L(p-1)}\right]^{\mathcal{T}}
$$

and

$$
\omega=e^{-j\left(2 \pi / N_{c}\right)} .
$$

With the above frequency domain formulation, partly following the analysis strategy for LDC-CP-OFDM in [6], the diversity properties of LDC-CP-SCM are analyzed. Consider a pair of matrices $\mathbf{M}$ and $\tilde{\mathbf{M}}$ corresponding to two different blocks $\mathbf{C}$ and $\tilde{\mathbf{C}}$. Then the upper bound pairwise error probability between $\mathbf{M}$ and $\tilde{\mathbf{M}}$ is [17]

$$
P(\mathbf{M} \rightarrow \tilde{\mathbf{M}}) \leq\left(\begin{array}{c}
2 r-1 \\
r
\end{array}\right)\left(\prod_{a=1}^{r} \gamma_{a}\right)^{-1}(\rho)^{-r},
$$

where $r$ is the rank of $\boldsymbol{\Lambda}=(\mathbf{M}-\tilde{\mathbf{M}}) \mathbf{R}_{\mathbf{H}}(\mathbf{M}-\tilde{\mathbf{M}})^{\mathcal{H}}$ and $\mathbf{R}_{\mathbf{H}}=\mathbb{E}\left\{\mathbf{H}[\mathbf{H}]^{\mathcal{H}}\right\}$ is the correlation matrix of $\mathbf{H}, \gamma_{a}, a=$ $1, \cdots, r$, are the non-zero eigenvalues of $\boldsymbol{\Lambda}$.

Then the corresponding rank and product criteria are as follows:

1. Rank criterion: The minimum rank of $\boldsymbol{\Lambda}$ over all pairs of different frequency domain symbol matrices $\mathbf{M}$ and $\tilde{\mathbf{M}}$ should be as large as possible.

2. Product criterion: The minimum value of the product $\prod_{a=1}^{r} \gamma_{a}$ over all pairs of different frequency domain symbol matrices $\mathbf{M}$ and $\tilde{\mathbf{M}}$ should be maximized.

We remark that we can obtain a sufficient condition for LDC-CP-SCM to achieve full available joint frequency and time diversity in the channels, which is provided in

Theorem 1. 1. The necessary and sufficient condition to ensure $\operatorname{rank}(\mathbf{M}-\tilde{\mathbf{M}})=N_{C} T$ is

$$
\left[\mathbf{F}_{N c}\left(\mathbf{x}_{S C}^{(k)}-\widetilde{\mathbf{x}}_{S C}^{(k)}\right)\right]_{p, 1} \neq 0, k=1, \ldots, T, p=1, \ldots, N_{C} .
$$

2. In a LDC-CP-SCM system, the rank of $(\mathbf{M}-\tilde{\mathbf{M}})$ satisfies

$$
\operatorname{rank}(\mathbf{M}-\tilde{\mathbf{M}})=N_{C} T \text {. }
$$

a. The LDC-CP-SCM system achieves full available diversity order in the frequency selective channels, i.e. $\operatorname{rank}(\mathbf{\Lambda})=\operatorname{rank}\left(\mathbf{R}_{\mathbf{H}}\right)$.

$b$. The corresponding product design criterion for 
$L D C-C P$-SCM block is that the minimum of the products

$$
\Delta=\prod_{k=1}^{T} \prod_{p=1}^{N_{C}}\left|\left[\mathbf{F}_{N C} \mathbf{x}_{S C}^{(k)}\right]_{p, 1}-\left[\mathbf{F}_{N C} \widetilde{\mathbf{x}}_{S C}^{(k)}\right]_{p, 1}\right|^{2}
$$

taken over all pairs of distinct frequency domain symbol matrices $\mathbf{M}$ and $\tilde{\mathbf{M}}$ must be maximized.

3. Assume that the frequency selective channel order $L$ is constant over time. A condition for LDC-SCM to achieve available full joint frequency and time diversity order $r_{d}=\operatorname{rank}\left(\mathbf{R}_{\mathbf{H}}\right)$ is that there always exist $(L+1)$ indices, $1 \leqslant p^{(k)}=p_{1}^{(k)}, \ldots, p_{L+1}^{(k)} \leqslant N_{C}$, for each $k=$ $1, \ldots, T$ such that

$$
\left[\mathbf{F}_{N c}\left(\mathbf{x}_{S C}^{(k)}-\widetilde{\mathbf{x}}_{S C}^{(k)}\right)\right]_{p^{(k), 1}} \neq 0 .
$$

Note that this condition is a sufficient and necessary condition for frequency diversity and a sufficient condition for time diversity.

The proof is provided in the Appendix. Note that single-carrier systems are inherently able to achieve some frequency diversity. However, full frequency diversity order is not guaranteed in conventional uncoded single-carrier communications systems, especially in uncoded CP-SCM systems, and the frequency coding gain may be further improved through careful design $[18,19]$. A LDC-SCM block is across multiple SCM blocks in block time varying channels, and the LDC-SCM system has potential to achieve joint frequency-time diversity order up to $T(L+1)$, which is the maximally achievable diversity order or full diversity order. Although the design strategy of LDC-SCM systems to support a certain order of frequency diversity is different from that of LDC-OFDM [6], we can obtain the following corollary on the relation between full joint frequency and time diversity LDC-CP-SCM and LDC-CP-OFDM.

Corollary 1. Assume that a LDC-CP-OFDM block, $\mathbf{C}_{L D C \_O F D M}$, with $N_{C}$ subcarriers and T OFDM blocks achieves full joint frequency and time diversity order. Before IFFT, the k-th OFDM block within the LDC-CP-OFDM block $\mathbf{C}_{L D C \_O F D M}$ is expressed as $\mathbf{x}_{\text {OFDM }}^{(k)}$, where $k=1, \ldots, T$ and $\mathbf{x}_{O F D M}^{(k)}=\left[x_{O F D M(1)}^{(k)}, \ldots, x_{O F D M\left(N_{C}\right)}^{(k)}\right]^{\mathcal{T}}$.

Then the $k$-th SCM block within a LDC-CP-SCM, $\mathbf{C}_{L D C \_S C M}$, can be designed as

$$
\mathbf{x}_{S C}^{(k)}=\left[\mathbf{F}_{N C}\right]^{\mathcal{H}} \mathbf{x}_{O F D M}^{(k)},
$$

where $k=1, \ldots, T$ and $\mathbf{x}_{S C}^{(k)}=\left[x_{S C(1)}^{(k)}, \ldots, x_{S C\left(N_{C}\right)}^{(k)}\right]^{\mathcal{T}}$. The consequence is that this LDC-CP-SCM achieves full joint frequency and time diversity order in the time varying frequency selective channel.

Corollary 1 provides a method for constructing full joint frequency and time diversity LDC-CP-SCM. However, since the IFFT is involved, this LDC-CP-SCM construction is the same as LDC-CP-OFDM with IFFT processing, one may be concerned with related problems, such as high PAPR.

\section{Performance}

\subsection{Simulation setup}

Perfect channel knowledge is assumed at the receiver but not at the transmitter. Unless specifically mentioned, the number of data symbols per SCM block, $N_{C}$, is 32 . Two LDC constructions are considered in the simulations

1. U-LDC and HH-LDC: For $T \leq M$, uniform linear dispersion codes (U-LDC) [20] is defined as below: Denote $\mathcal{D}_{K}=\operatorname{diag}\left(1, e^{j \frac{2 \pi}{K}}, \ldots, e^{j \frac{2 \pi(K-1)}{K}}\right)$. Denote $\Pi_{K}$ as a matrix of size $K \times K$ with zeros except $\left[\Pi_{K}\right]_{a, a-1}=1, a=2, \ldots, K$ and $\left[\Pi_{K}\right]_{K, K}=1$. Denote $\mathbf{X}=\left[\mathbf{I}_{T}, \mathbf{Z}_{T \times(M-T)}\right]$ where $\mathbf{Z}_{T \times(M-T)}$ is a zero matrix. The $T \times M$ U-LDC dispersion matrices are:

$$
\mathbf{A}_{M(k-1)+l}=\mathbf{B}_{M(k-1)+l}=\frac{1}{\sqrt{T}}\left[\mathcal{D}_{T}\right]^{k-1} \boldsymbol{\Gamma}\left[\boldsymbol{\Pi}_{M}\right]^{l-1},
$$

where $k=1, \ldots, T$ and $l=1, \ldots, M$. In the case of $T=$ $M$, U-LDC becomes the HH square code as described in (31) of [9].

2. LCP-LDC and FTLCP-LDC: The $Q \times Q$ LCP-LDC [6] encoding matrix could be constructed using linear constellation precoding design A (LCPA) [21,22] as

$$
\mathbf{G}_{L C P-L D C}=\Theta=\left[\begin{array}{cccc}
1 & \alpha_{1} & \cdots & \alpha_{1}^{Q-1} \\
1 & \alpha_{2} & \cdots & \alpha_{1}^{Q-1} \\
\vdots & \vdots & \ddots & \vdots \\
1 & \alpha_{Q} & \cdots & \alpha_{Q}^{Q-1}
\end{array}\right]
$$

where $Q=N_{F} T$, and $\left\{\alpha_{q}, q=1, \ldots, Q\right\}$ are defined in $[21,22]$. One encoding matrix of LTC considered in the simulations is also LCPA.

We propose the encoding matrix of a Fourier Transformed LCP-LDC(FTLCP-LDC) as

$$
\mathbf{G}_{T L C P-L D C}=\mathbf{U G}_{T L C P-L D C},
$$

where $\mathbf{U}=\operatorname{diag}\left(\mathbf{F}_{N_{F}}, \ldots, \mathbf{F}_{N_{F}}\right)$.

The LDC or LTC symbol coding rates of the proposed systems used in simulations are all unity. Compared with uncoded CP-SCM systems, no bandwidth is lost unless forward error control is used. The sizes of all LDC codewords are identically $T \times N_{F}$. Unless specifically mentioned, an evenly spaced mapping either from LDC to channel data symbol index for LDC-CP-SCM or from LDC to subcarrier index for LDC-CP-OFDM is used in simulations.

The frequency selective channel has $(L+1)$ paths exhibiting an exponential power delay profile, and the guard interval size of each SCM block is set to $N_{g}=L$. Source data symbols use QPSK modulation in all simulations. 


\subsection{Comparison between LDC-SCM and SCM systems}

Fig. A. 2 shows the diversity performance comparison of bit error rate (BER) vs. SNR between LDC-CP-SCM (LDCCP-SCM and LDC-ZP-SCM) and uncoded CP-SCM under the setting of $N_{F}=16$ and choosing HH-LDC. Similarly, the comparisons of LDC-ZP-SCM and uncoded ZP-SCM are shown.

When CCI is a multiple of $T$, i.e. $C C I=16$, the effects of time diversity in the channels are removed, and it can be observed that the performances of LDC-SCM and SCM are quite similar, which suggests that the LDC-SCM systems using the chosen LDC do not provide notable frequency coding improvement over SCM systems.

When CCI is not a multiple of $T$, i.e. $C C I<16$, clearly, BER performance of LDC-SCM is remarkably better than that of both uncoded SCM, which is primarily attributed to time diversity. Time diversity order is maximized only if the channel provides block-wise temporal independence. As shown in Fig. A.2, the performance of LDCSCM systems is significantly influenced by channel dynamics, i.e., time correlation. This indicates that LDC-SCM effectively exploits available temporal diversity.

6.3 Comparison of LDC-ZP-SCM and ZP-SCM under MMSE vs. low complexity MMSE receivers

Fig. A. 3 shows the comparison of LDC-ZP-SCM and ZPSCM using MMSE-TDE and MMSE-LC-ADFE. Due to the layered TSE structure discussed in Section 4, it can been seen that, even at low complexity, LDC-ZP-SCM using MMSE-LC-ADFE performs close to that using MMSE TDE over the entire SNR range. At a BER of $10^{-3}$, LDCZP-SCM using low complexity MMSE equalizer results in only $0.4 \mathrm{~dB}$ performance degradation.

\subsection{Comparison between LDC-CP-SCM and LDC-CP- OFDM systems}

In this subsection, we test coded system performance using maximum likelihood decoding, and set $N_{C}=N_{F}=8$ and $T=2$. Fig. A. 4 shows a diversity performance comparison of BER vs. SNR between LDC-CP-SCM and LDC-CPOFDM. The design cases of $C C I=1$ significantly outperforms the design cases of $C C I=2$. The best performance of two optimal cases, LDC-OFDM (LCP-LDC) and LDCSCM (FTLCP-LDC) perform similarly, which agrees with the result in Corollary 1. As shown in Fig. A.4, simply applying LCP-LDC in SCM systems does not obtain desirable performance. However, the performance of U-LDC in SCM systems is acceptable.

6.5 Comparison of cyclic-prefix (CP) based systems under $\mathrm{CFO}$ effects

In Fig. A.5, the detrimental effects of CFO are observed.
Under the normalized CFO setting of $\varepsilon=0.02$, CP based systems without CFO outperform those with CFO, especially at higher SNRs. In higher SNRs, the performance loss of LDC-CP based systems due to CFO effects is higher than that of uncoded $\mathrm{CP}$ based systems. Although having the highest performance loss under CFO effects, LDC-CP$\mathrm{SCM}$ has the best performance in time varying frequency selective channels.

\subsection{Comparison between LDC-CP-SCM and LTC-CP- SCM systems}

Fig. A. 6 shows a diversity performance comparison of BER vs. SNR between LDC-CP-SCM and LTC-CP-SCM. In all curves shown, LDC-CP-SCM (using HH-LDC) perform similarly to LTC-CP-SCM (using LCPA), which suggests that LTC-CP-SCM (using LCPA) is close full joint frequency-time diversity design.

It is deserved to remark that compared to general LDCSCM, LTC-SCM has much lower complexity, which agrees with the design principle of SCM-FDE for mobile terminal or uplink transmission.

\section{Conclusions}

This paper proposes the use of high-rate LDC in singlecarrier block communications systems with either cyclicprefix or zero-padding guard intervals in time-varying frequency selective channels. This paper also provides a sufficient condition for LDC-CP-SCM to maximize all available joint frequency and time diversity gain and coding gain. This paper provides the relation between LDC-OFDM and LDC-SCM of full joint frequency-time diversity designs. Simulations reveal that LDC-SCM may outperform both uncoded SCM in time-varying frequency selective channels, even under CFO effects. LDC-ZP-SCM may be implemented using low complexity MMSE equalizers without significant performance degradation. Finally, this paper also proposes a low complexity LDC-SCM design, LTC-SCM, which is able to have performance close to that of joint full frequency-time diversity design.

\section{Appendix: Proof of Theorem 1}

Proof. 1) Note that $\mathbf{M}-\tilde{\mathbf{M}}$ is a diagonal matrix. Thus, to maximize rank of $\mathbf{M}-\tilde{\mathbf{M}}$, the necessary and sufficient condition is that all the diagonal elements are non-zero. Thus, to ensure $\operatorname{rank}(\mathbf{M}-\tilde{\mathbf{M}})=N_{C} T$, it is necessary and sufficient to have $c_{p}^{(k)}-\widetilde{c_{p}^{(k)}} \neq 0, p=1, \ldots, N_{C}, k=$ $1, \ldots, T$.

Recall $c_{p}^{(k)}=\left[\mathbf{z}_{C P-S C}^{(k)}\right]_{p, 1}$ and $\mathbf{z}_{C P-S C}^{(k)}=\mathbf{F}_{N C} \mathbf{x}_{S C}^{(k)}$, where $p=1, \ldots, N_{C}$ and $k=1, \ldots T$.

Hence, the necessary and sufficient condition is

$$
\left[\mathbf{z}_{C P-S C}^{(k)}-\widetilde{\mathbf{z}}_{C P-S C}^{(k)}\right]_{p, 1}=\left[\mathbf{F}_{N C}\left(\mathbf{x}_{S C}^{(k)}-\widetilde{\mathbf{x}}_{S C}^{(k)}\right)\right]_{p, 1} \neq 0,
$$

where $p=1, \ldots, N_{C}$ and $k=1, \ldots T$. 
2) Note that the condition is that the rank of frequency domain matrix satisfies $\operatorname{rank}(\mathbf{M}-\tilde{\mathbf{M}})=N_{C} T$. Using the relevant derivation strategy used in [6], we get

$$
\begin{aligned}
& \operatorname{rank}(\mathbf{\Lambda})=\operatorname{rank}\left((\mathbf{M}-\tilde{\mathbf{M}}) \mathbf{R}_{\mathbf{H}}(\mathbf{M}-\tilde{\mathbf{M}})^{H}\right) \\
& =\operatorname{rank}\left(\mathbf{R}_{\mathbf{H}}(\mathbf{M}-\tilde{\mathbf{M}})^{\mathcal{H}}\right) \\
& =\operatorname{rank}\left(\mathbf{R}_{\mathbf{H}}\right)
\end{aligned}
$$

where

$$
\begin{aligned}
& \mathbf{R}_{\mathbf{H}}=E\left\{\left(\mathbf{I}_{T} \otimes \mathbf{W}\right) \mathbf{h}\left[\left(\mathbf{I}_{T} \otimes \mathbf{W}\right) \mathbf{h}\right]^{\mathcal{H}}\right\} \\
& =\left[\mathbf{I}_{T} \otimes \mathbf{W}\right] \mathbf{\Phi}\left[\mathbf{I}_{T} \otimes[\mathbf{W}]^{\mathcal{H}}\right],
\end{aligned}
$$

where $\boldsymbol{\Phi}=E\left\{\mathbf{h}[\mathbf{h}]^{\mathcal{H}}\right\}, \mathbf{W}=\left[\mathbf{w}_{1}, \cdots, \mathbf{w}_{N_{C}}\right]^{\mathcal{T}}$, and $\mathbf{h}=$ $\left[\left[\mathbf{h}^{(1)}\right]^{\mathcal{T}}, \cdots,\left[\mathbf{h}^{(T)}\right]^{\mathcal{T}}\right]^{\mathcal{T}}$.

The frequency domain symbols in LDC-SCM are the results of size $N_{C}$ Fourier transformation of source symbols, and thus the size of $\mathbf{M}$ must be $N_{C} T \times N_{C} T$. Note that the size of frequency-time block $\mathbf{M}^{(i)}$ of LDC-OFDM is $T N_{F(i)} \times T N_{F(i)}$, where $N_{F(i)}$ is usually much less than $N_{C}$.

Further, the product design criterion for CP-SCCB is that the minimum of products,

$$
\begin{aligned}
& \Delta=\prod_{k=1}^{T} \prod_{p=1}^{N_{C}}\left(\left|c_{p}^{(k)}-\widetilde{c}_{p}^{(k)}\right|^{2}\right) \\
& =\prod_{k=1}^{T} \prod_{p=1}^{N_{C}}\left|\left[\mathbf{F}_{N C} \mathbf{x}_{S C}^{(k)}\right]_{p, 1}-\left[\mathbf{F}_{N C} \widetilde{\mathbf{x}}_{S C}^{(k)}\right]_{p, 1}\right|^{2},
\end{aligned}
$$

taken over distinct codewords must be maximized.

\section{Acknowledgement}

This work was supported in part by Natural Sciences and Engineering Research Council of Canada (NSERC) Discovery Grant 41731. This work was supported in part by the National Basic Research Program of China (973 Program No. 2012CB316100) and the National Natural Science Foundation of China (NSFC) under grant No.60872013/61032002.

\section{References}

[1] D. Falconer, S.L. Ariyavisitakul, A. Benyamin-Seeyar, and B. Eidson, "Frequency domain equalization for single-carrier broadband wireless systems," IEEE Commun. Mag., pp.58-66, Apr. 2002.

[2] R.A. Litis, "Iterative joint decoding and sparse channel estimation for single-carrier modulation," Proc. IEEE Int. Conf. on Acoustics, Speech and Signal Processing, pp.2689-2692, Mar. 2008.

[3] Y.R. Zheng and C. Xiao, "Channel estimation for frequency-domain equalization of single-carrier broadband wireless communications," IEEE Trans. Vehicular Technology, vol.58, no.2, pp.815-823, Feb. 2009.
[4] J. Heiskala and J. Terry, OFDM wireless LANs: a theoretical and practical guide, SAMS, 2002.

[5] T. Buzid, S. Reinhardt, and M. Huemer, "A comparision of OFDM and non-linear SC/FDE signals: Non-linear SC/FDE signals: nonlinear amplification," Proc. European Wireless Conf. 2005, Apr. 2005.

[6] J. Wu and S.D. Blostein, "High-rate diversity across time and frequency using linear dispersion," IEEE Trans. Commun., vol.56, no.8, pp.1469-1477, Sept. 2008.

[7] Z. Wang, X. Ma, and G.B. Giannakis, "OFDM or single-carrier zero padded block transmissions," Proc. IEEE Wireless Commun. and Networking Conference (WCNC), Orlando, FL, pp.660-664, Mar. 2002.

[8] B. Muquet, Z. Wang, G.B. Giannakis, M. de Courville, and P. Duhamel, "Cyclic prefixing or zero padding for wireless multicarrier transmissions?," IEEE Trans. Commun., vol.50, no.12, pp.2136-2148, Dec. 2002.

[9] B. Hassibi and B.M. Hochwald, "High-rate codes that are linear in space and time," IEEE Trans. Inform. Theory, vol.48, no.7, pp.18041824, July 2002.

[10] J. Wu and S.D. Blostein, "Linear dispersion over time and frequency," Proc. IEEE ICC 2004, pp.254-258, June 2004.

[11] H.V. Poor, An introduction to signal detection and estimation, Springer Verlag, Mar. 1994.

[12] M.O. Damen, H.E. Gamal, and N.C. Beaulieu, "Linear threaded algebraic space-time constellation," IEEE Trans. Inform. Theory, pp.2372-2388, Oct. 2003.

[13] P. Dayal and M.K. Varanasi, "Maximal diversity algebraic spacetime codes with low peak-to-mean power ratio," IEEE Trans. Inform. Theory, vol.51, no.5, pp.1691-1708, May 2005.

[14] U. Tureli, P.J. Honan, and H. Liu, "Low-complexity nonlinear least squares carrier offset estimator for OFDM: identifiability, diversity and performance," IEEE Trans. Sig. Proc., vol.52, no.9, pp.24412452, Sept. 2004.

[15] J.A. Fessler and A.. Hero, "Space-alternating generalized expectationmaximization algorithm,” IEEE Trans. Sig. Proc., vol.42, no.10, Oct. 1994.

[16] T. Someya and T. Ohtsuki, "SAGE algorithm for channel estimation and data detection with tracking the channel variation in MIMO system," Proc. IEEE Globecom, Nov. 2004.

[17] S. Siwamogsatham, M.P. Fitz, and J.H. Grimm, "A new view of performance analysis of transmit diversity schemes in correlated Rayleigh fading," IEEE Trans. Inform. Theory, vol.48, no.4, pp.950956, Apr. 2002.

[18] Z. Liu, "Maximum diversity in single-carrier frequency-domain equalization,” IEEE Trans. Inform. Theory, vol.51, no.8, pp.29372940, Aug. 2005.

[19] W. Zhang, "Comments on "Maximum diversity in single-carrier frequency-domain equalization"," IEEE Trans. Inform. Theory, vol.52, no.3, pp.1275-1277, Mar. 2006.

[20] J. Wu and S. D.Blostein, "Rectangular information lossless linear dispersion codes," IEEE Trans.Wireless Commun., vol.9, no.2, pp.517-522, Feb. 2010.

[21] Y. Xin, Z. Wang, and G.B. Giannakis, "Space-time diversity systems based on linear constellation precoding," IEEE Trans. on Wireless Commun., vol.2, pp.294-309, Mar. 2003.

[22] Z. Liu, Y. Xin, and G.B. Giannakis, "Linear constellation precoded OFDM with maximum multipath diversity and coding gains," IEEE Trans. Commun., vol.51, no.3, pp.416-427, Mar. 2003. 


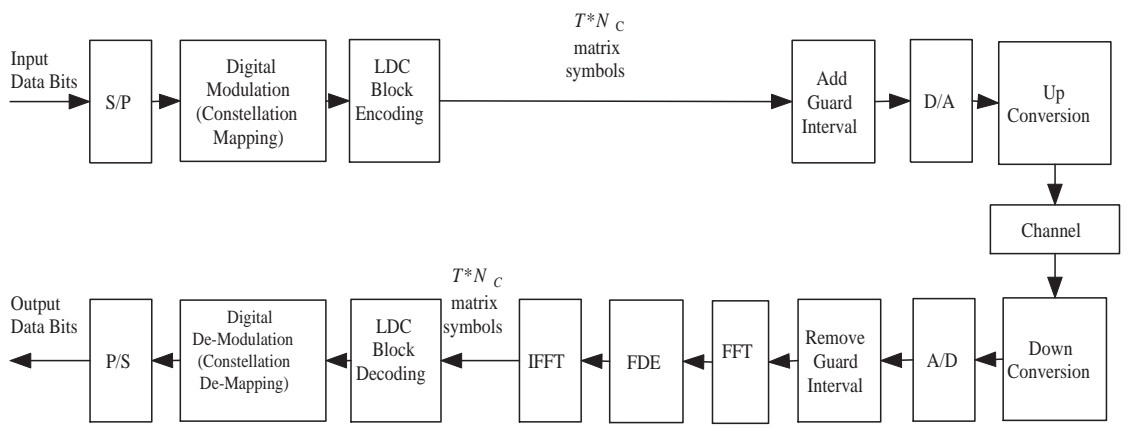

Fig. A. 1 Proposed LDC-CP-SCM system model

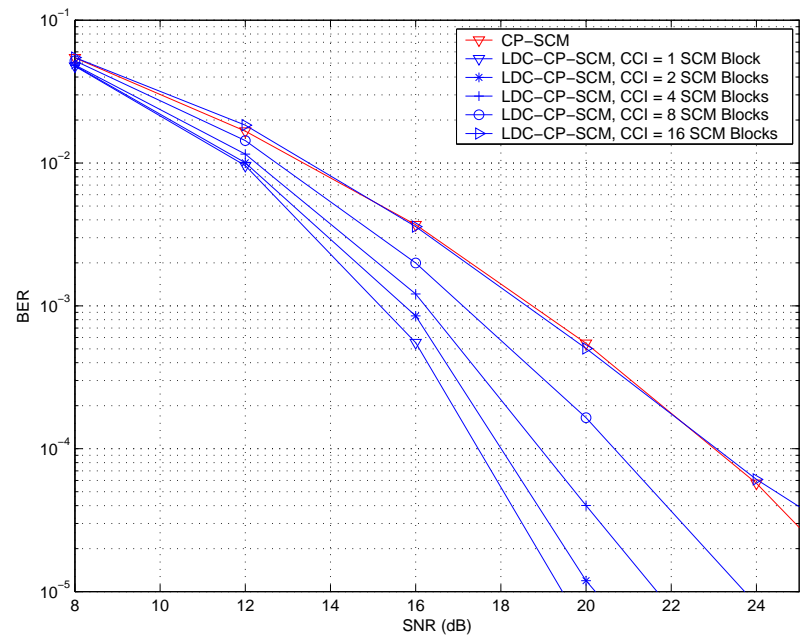

Fig. A. 2 BER Performance of LDC-CP-SCM (using HH-LDC) vs. CPSCM using MMSE-FDE, $N_{C}=32, N_{F}=16, T=16, L=4$

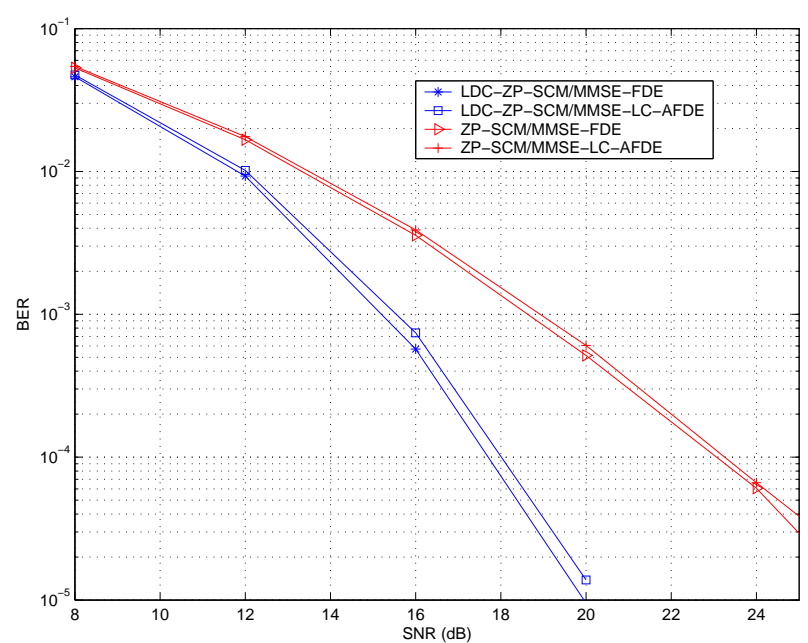

Fig. A. 3 BER Performance of LDC-ZP-SCM (using HH-LDC) using MMSE-FDE vs. MMSE-LC-AFDE, channel order $=3, C C I=1 \mathrm{SCM}$ block, $N_{C}=32, N_{F}=8, T=8$

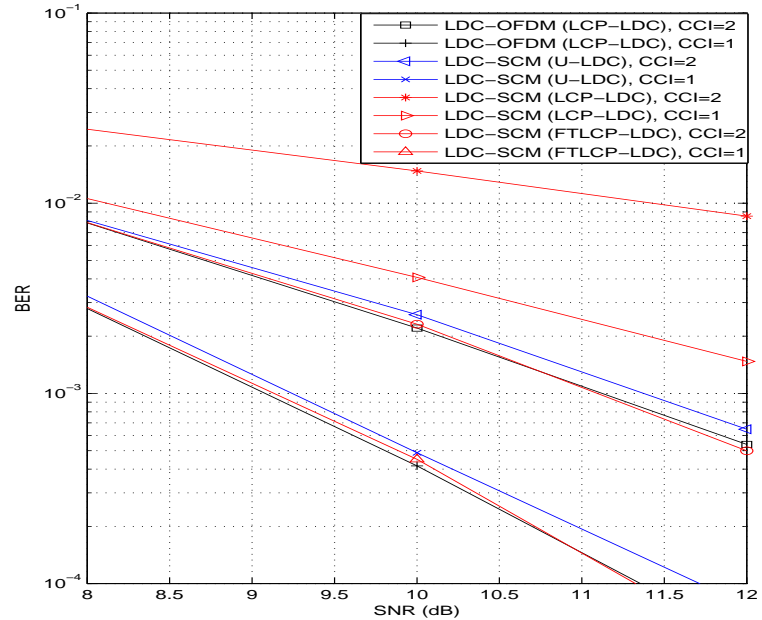

Fig. A. 4 BER Performance of LDC-CP-SCM vs. LDC-CP-OFDM, $L=$ $2, N_{C}=N_{F}=8, T=2$

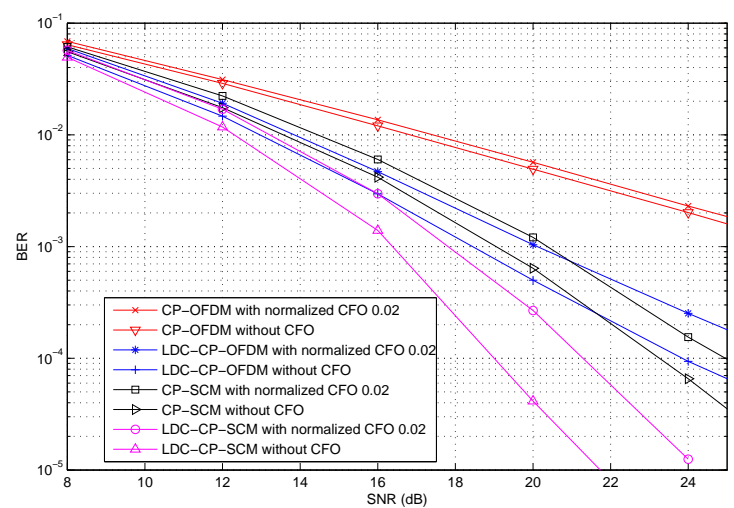

Fig. A. 5 BER Performance of CP based systems using MMSE-FDE under CFO effects (using HH-LDC), $L=2, N_{C}=32, N_{F}=16, T=16$, $F S R=2, C C I=2$ (OFDM or SCM blocks) 


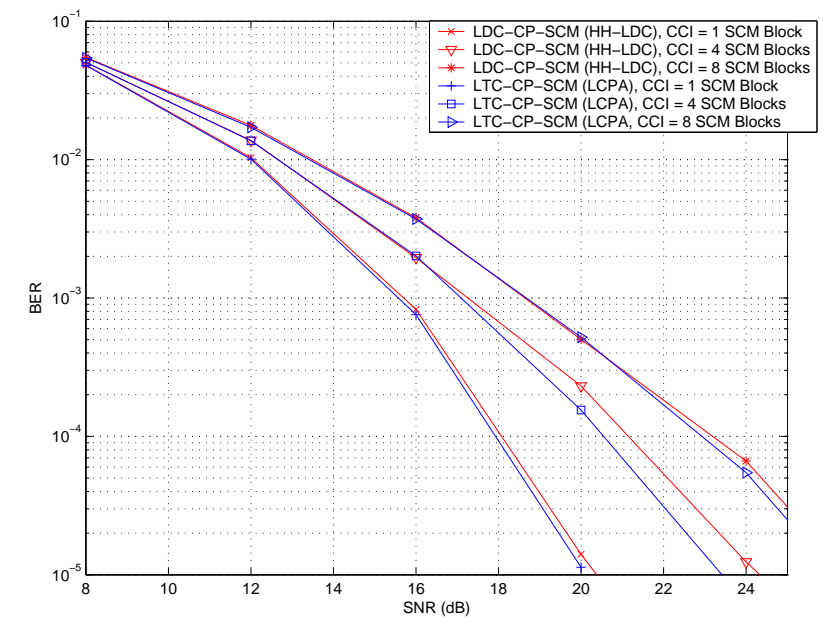

Fig. A. 6 BER Performance of LDC-CP-SCM (using HH-LDC) vs. LTC-CP-SCM (using LCPA), $L=6, N_{C}=32, N_{F}=8, T=8$

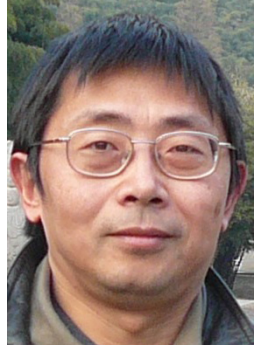

Jinsong Wu He obtained Ph.D. degree in electrical engineering from Queen's University, Kingston, Canada in 2006. Since October 2010, he has worked as Research Scientist in Bell Laboratories, Shanghai, China. He has experienced research and development positions relevant to communications engineering in Nortel Networks Canada, Philips Research USA, and Sprint-Nextel USA. He is the Editorin-chief of the comprehensive CRC Press book, entitled "Green Communications: Theoretical Fundamentals, Algorithms, and Applications," to be published in 2012. His broad research interests lie in sustainable and green communications and computing, information theory and communication signal processing, data networking, and communications applications and services. He has served as technical program committee members in more than 24 key international telecommunications relevant conferences. He is the proposer and Moderator/Chair of the Technical Panel on Green Communications and Computing in IEEE INFOCOM 2012, IEEE ICC 2012, and IEEE GLOBECOM 2012. $\mathrm{He}$ is the Founding Chair of Technical Subcommittee on Green Communications and Computing (TSCGCC), IEEE Communications Society. He has served as the Vice-Chair, Track on Green Networks and Communication Systems, the Selected Areas in Communications Symposium, IEEE GLOBECOM 2012. He is one of Technical Program Committee Chairs, the 2012 IEEE Online Conference on Green Communications. He currently is an IEEE Senior Member. Email: wujs@ieee.org

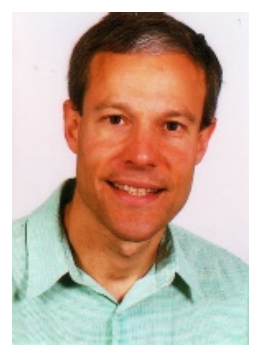

Steven D. Blostein (MIEEE '88, SMIEEE 96) He received his B.S. degree in Electrical Engineering from Cornell University, Ithaca, NY, in 1983, and the M.S. and Ph.D. degrees in Electrical and Computer Engineering from the University of Illinois, Urbana-Champaign, in 1985 and 1988, respectively. He has been on the faculty in the Department of Electrical and Computer Engineering Queen's University since 1988 and currently holds the position of Professor. From 2004-2009 he was Department Head. He has also been a consultant to industry and government in the areas of image compression, target tracking, radar imaging and wireless communications. His current interests lie in the application of signal processing to problems in wireless communications systems, including synchronization, network MIMO and physical layer optimization for multimedia transmission. He has been a member of the Samsung 4G Wireless Forum as well as an invited distinguished speaker. He served as Chair of IEEE Kingston Section (1994), Chair of the Biennial Symposium on Communications $(2000,2006,2008)$, Associate Editor for IEEE Transactions on Image Processing (1996-2000), and Publications Chair for IEEE ICASSP 2004. For a number of years has been serving on Technical Program Committees for IEEE Communications Society conferences that include ICC, Globecom and WCNC. He has been serving as an Editor of IEEE Transactions on Wireless Communications since 2007. He is a registered Professional Engineer in Ontario and a Senior Member of IEEE and a Member of IEICE, Email: steven.blostein@queensu.ca.

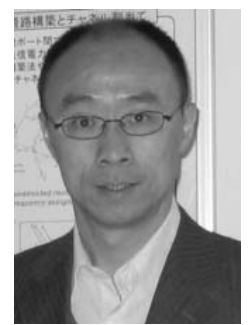

Qingchun Chen He received his Ph.D. degree from Southwest Jiaotong University in 2004, his B.S. and M.S. degrees from Chongqing University in 1994 and 1997, respectively. He has been with the Institute of Mobile Communication and the Key Lab of Information Coding \& Transmission, Southwest Jiaotong University since 2004, where he is now the 
full professor. He is the inventor of 7 patents and the author of over 60 research papers. His research interests include: information theory \& coding, collaborative communication, compressive sensing and wireless network technologies. Contact information: the provincial key lab of information coding and transmission, Southwest Jiaotong University, Chengdu, Sichuan, 610031, P.R. China, E-mail: qcchen@ home.swjtu.edu.cn.

Pei Xiao He received the $\mathrm{PhD}$ degree in Electrical Engineering from Chalmers University of Technology, Sweden, in 2004. He held positions at Nokia Networks in Tampere, Finland from 1997 till 2000. Following his $\mathrm{PhD}$ graduation, he worked as a research fellow at Newcastle University and Queen's University Belfast. He has been with the Centre for Communication Systems Research (CCSR), University of Surrey as a lecturer since January 2011. Dr. Pei Xiao is a senior member of IEEE and member of IEICE. He has published over 100 papers including 35 journal articles and 5 book chapters. His research interests and expertise span a wide range of areas in communications theory and signal processing for wireless communications. Email: P.Xiao@surrey.ac.uk 